\title{
Analysis of YouTube as a Source of Information for West Nile Virus Infection
}

\author{
Divyanshu Dubey, MD; Amod Amritphale, MD; Anshudha Sawhney, MBBS;
}

Devashish Dubey, MBBS; and Nupur Srivastav, MBBS

\begin{abstract}
Introduction: A major outbreak of West Nile Virus was seen last year in different parts of the United States. Adequate dissemination of correct information about the disease would have helped decrease its spread and the associated panic in the general population. In this study, we looked into the use of YouTube as a resource for providing information about West Nile Virus infection.
\end{abstract}

Objective: This study aims to identify and evaluate YouTube as resource for providing information on West Nile Virus infection to the general public.

Methods: YouTube was searched on November 25, 2012, using the keywords West Nile Virus epidemic, West Nile Virus infection, and West Nile Virus prevention for videos uploaded in the past 6 months containing relevant information about the disease. The videos were classified as useful, misleading, or as news updates based on the type of information contained. Total viewership, number of days since upload, total duration of videos, and source of upload were noted.

Results: A total of 106 videos with information on West Nile Virus infection were included in the study, with $79.24 \%$ having useful information about the disease. Among the useful videos, 5I/84 $(60.71 \%)$ had information on disease prevention, and 29/84 (34.52\%) contained information on news and research updates. The majority of these videos were uploaded by individuals (54.6\%) or news agencies (4I.8\%). Healthcare agencies contributed only $3.4 \%$ of the total videos. Even though the useful videos represented $72 \%$ of all videos, there was significantly higher total viewership and viewership per day for the non-useful videos $(P<0.05)$.

Conclusions: YouTube may be a significant resource for dissemination of information on public health issues like West Nile virus infection and should be targeted by healthcare agencies for this use. The major drawback of this medium is lack of verification by authorized healthcare professionals before these videos are made available for viewing by the community. Hence, a strict caution should be exercised in obtaining the information from unauthorized videos posted on YouTube.

Keywords: West Nile Virus; YouTube; Infection

$\mathrm{T}$ 4,531 cases and 183 fatalities in the United States (US) is one of the worst outbreaks of the decade. It was second only to the epidemic in 2003 that saw more than 9,000 cases and 265 fatalities in the US. During 2012, the WNV epidemic had spread to several countries in Europe including Israel, Greece, and Italy. ${ }^{1,2}$ As of July 30, 2013, a total of 53 cases of WNV disease had been reported to the Centers for Disease
Corresponding Author: Divyanshu Dubey, MD; University of Texas Southwestern Medical Center; Department of Neurology; 5323 Harry Hines Boulevard; Dallas, TX 75235; Tel: (2I4) 283-9782; Fax: (2I4) 283-9782; Email: divyanshudubey87@gmail.com
Control (CDC), including 3 deaths. Nearly $40 \%$ of the cases were classified as neuroinvasive disease (such as meningitis or encephalitis), and $60 \%$ were classified as nonneuroinvasive disease. ${ }^{1}$ Resurgence of this arboviral disease emphasizes the importance of outbreak prevention measures, as well as awareness and preparedness of the general public.

Received: August 21, 2013

Revised: October 15, 2013

Accepted: November 14, 2013

doi: $10.3121 / \mathrm{cmr} .2013 .1194$ 
Dissemination of accurate information is important to encourage people to be compliant with prevention guidelines and practice-specific behaviors for preventing spread of the disease. Effective measures for tackling WNV infection include identifying the diseased state, providing supportive measures, preventing longterm complications associated with the illness, and educating the public about the disease. Awareness about the clinical symptomatology can lead to early presentation to the appropriate medical facilities, subsequently leading to decrease in disease complications and mortality.

The Internet has become one of the most powerful sources of healthcare information for the general public. Approximate viewership of 100 million per day is a marker of the immense popularity and easy accessibility of YouTube among the masses. ${ }^{4,5}$ Information regarding a multitude of medical conditions through the means of videos is easily provided to the general public. However, the potential risk of posting misleading information along with a lack of verification could adversely influence the perception of those viewing the videos. This emphasizes the importance of analysis and verification of the information posted and available on YouTube. In the past, YouTube had been evaluated as a source of information on vaccination, ${ }^{6,7}$ tobacco use, ${ }^{8}$ breast feeding, ${ }^{9} \mathrm{H} 1 \mathrm{~N} 1,{ }^{10}$ kidney stones, ${ }^{11}$ basic life support, cardiopulmonary resuscitation, ${ }^{12}$ Tourette's syndrome, ${ }^{13}$ and Epley's maneuver. ${ }^{14}$

The use of YouTube as a source of information for a WNV epidemic has never been previously evaluated. In this study we have evaluated the use of YouTube by health organizations, independent users, and news agencies to disseminate information during the WNV outbreak from the months of June through November 2012.

\section{Methods}

YouTube was searched on November 25, 2012, using the keywords West Nile Virus epidemic, West Nile Virus infection, and West Nile Virus prevention for videos uploaded in the past 6 months containing information about the disease. Duplicated videos were not included in the study. The videos were analyzed by two independent resident physicians and classified as useful, misleading, or as news updates based on the information provided. Useful videos were defined as those containing scientifically relevant information about symptoms, disease prevention, measures taken to combat the disease, or any one of these. Misleading/non-useful videos were those that contained scientifically unproven information. Updates were videos uploaded by health agencies, news agencies, or independent users quoting the CDC website with information about the current status of the disease in terms of mortality and extent of spread only, devoid of other useful information regarding primary or secondary prevention of the disease or its complications.

The degree of agreement between the two researchers was determined by kappa co-efficient of agreement. Videos were also categorized into three groups according to their source: National agencies (like CDC), news agencies, and independent users. Total viewership, number of days since upload, and total duration of videos were analyzed. Assessment of the videos for providing information regarding disease prevention and symptomatic manifestation of infection was also conducted. Data entry and analysis were done using SPSS, version 19.0, software. An independent $t$ test was applied to compare the means of continuous variables. A $P$ value of $<0.05$ was considered significant.

\section{Results}

A total of 106 videos pertaining to the WNV epidemic were

Table 1. Detailed characteristics of various categories of YouTube videos with relevant information about the West Nile Virus 2012 epidemic in the United States.

$\left.\begin{array}{lll}\hline & \begin{array}{l}\text { Useful videos } \\ \text { (correct information and updates) }\end{array} & \begin{array}{l}\text { Non-useful videos } \\ \text { (misleading videos) }\end{array} \\ \hline \text { Number of videos (\%) } & 84(79.24 \%) & 22(20.76 \%) \\ \text { Total duration (minutes [\%]) } & 206.54004(72.41 \%) & 78.680008(27.58 \%) \\ & & 3.22\end{array}\right)$

CDC, Centers for Disease Control; SD, standard deviation. 


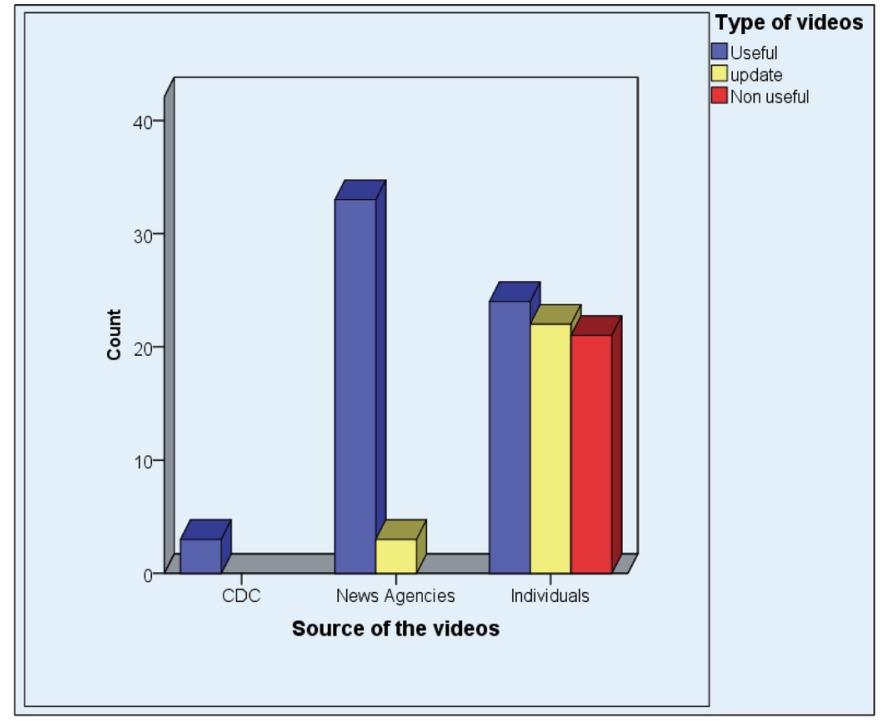

Figure 1: Graph depicting the contribution of various sources based on the types of videos uploaded.

evaluated by two independent physicians. Kappa coefficient of agreement between the physicians was 0.95 . A total of $79.24 \%$ (84) videos were found to be useful. It was determined that $\mathrm{CDC}$ and other national agencies had contributed $3.4 \%$ (3) of videos, news agencies contributed $41.8 \%$ (36) of videos, while the majority of the videos were contributed by individuals 54.6\% (67).

Of the total videos found to be useful, 23.8\% (25) videos provided updates on the disease trend and current prevalence in the community. A total of 58\% (49) of the useful videos had information regarding disease prevention, whereas $33.7 \%$ (28) had information regarding symptomatology of the disease. Misleading information was posted in $20.76 \%$ (22) videos. All such videos were contributed by individuals.

On further analysis of the selection of videos found to be useful, it was determined that these videos had a mean viewership of 228 per day and a mean duration since upload of 106 days (standard deviation $[\mathrm{SD}]=34.58$ ). There was a positive correlation between viewership per day and length of the videos $(\mathrm{r}=0.48 ; P<0.0001)$ and viewership per day and duration since upload $(\mathrm{r}=0.3 ; P<0.0001)$.

Mean viewership per day for useful videos was 2.20 $(\mathrm{SD}=5.78)$, whereas that for non-useful videos was $6.28(\mathrm{SD}=$ 5.89). The viewership for non-useful videos was significantly higher than useful videos. There was a gradual increase in the number of useful videos posted until the month of August, with a decline thereafter. The number of updates peaked in the initial months (June-July 2012) of the epidemic. Detailed characteristics of the various categories of YouTube videos is provided in table 1 and a graphic depiction of types of videos based on uploading agency is provided in figure 1.

\section{Discussion}

On the day of analysis (November 25, 2012), 79.24\% of the videos were found to be useful. The mean duration of the useful videos since upload was 106 minutes. The videos posted by national healthcare agencies contributed to a meager 3.4\% as compared to the videos posted by individuals and news agencies, which were $54.6 \%$ and $41.8 \%$, respectively. Given the enormous impact on healthcare caused by the WNV epidemic in the year 2012, there should be an increased number of videos posted by national healthcare agencies. The absence of videos posted by national healthcare agencies as sponsored links further adversely impacted the availability and accessibility of correct, useful, and comprehensive information to the general public.

There was significantly higher viewership of the non-useful videos $(P<0.05)$, and some of these videos portrayed misleading information. One such video suggested that the outbreak of the WNV infection was a government conspiracy to perform inspection of backyards on private property. Another video labeled the epidemic as a biological weapon trial. One video uploaded by an individual described the epidemic as a sign of alien warfare. Some suggested that this was a biblical prophecy being fulfilled or that the outbreak of the infection was secondary to the shift of the North Pole. Non-useful videos also presented misleading information about the aerial pesticide spraying, portraying it as a means of chemical warfare initiated by the government against the citizens. This further emphasizes the importance of verification and authentication of such videos by healthcare professionals.

Studies have been conducted in the past on several of topics of public interest. An analysis on the human papilloma virus (HPV) vaccination coverage found that three-fourths of the video clips $(74.9 \%)$ provided positive information on the HPV vaccine; ${ }^{6}$ whereas a paper on immunization revealed that as compared to useful videos, misleading videos were more likely to be viewed and receive a higher mean star rating. ${ }^{7}$ Pandey et $a l,{ }^{10}$ in their paper on the H1N1influenza pandemic, showed that the difference in viewership between useful and misleading videos was not statistically significant.

The magnitude of the 2012 WNV outbreak was hypothesized to be secondary to the presence of migratory birds combined with increased mosquito population and favorable weather conditions. ${ }^{15}$ An unusually mild winter, along with an early spring and hot summer in many parts of the US was considered to foster mosquito breeding. ${ }^{15}$

Various personal preventive strategies that were emphasized in the posted videos included eliminating stagnant water sources in the community, staying indoors during dusk and dawn, wearing long pants and long sleeves, having wellfitting screens on doors and windows, and using DEET (N,NDiethyl-meta-toluamide) insect repellent outdoors.

Due to the absence of specific antiviral medications and suitable human vaccines against WNV, preventive measures are of utmost importance in avoiding fatalities associated 
with the WNV infection. Hence, preventive strategies need to be emphasized well in advance before a major outbreak occurs in the coming years. These measures should include posting of authentic and verified measures on YouTube by national healthcare agencies.

\section{Limitations}

The study evaluated the videos posted only over a 6-month period. We evaluated the use of YouTube as a source of information only for the outbreak of 2012. Videos posted during the previous outbreaks were not evaluated. Our study was limited to a direct search of YouTube and did not analyze the YouTube videos accessed from other websites or posted links. Assessment of viewership could have been affected by accounting for repeated viewing from the same Internet Protocol address as multiple viewers. Only English language videos were analyzed in this study, but information could have been available in multiple other languages as well.

\section{Conclusions}

YouTube contains a significant amount of useful information on WNV infection. However, there is a scarcity of useful videos uploaded by health agencies like CDC or Red Cross. A major drawback of this medium is lack of verification by authorized healthcare professionals before these videos are made available for viewing by the community. Hence, a strict caution should be exercised in obtaining the information from unauthorized videos posted on YouTube.

\section{References}

1. Centers for Disease Control and Prevention. West Nile Virus. Available at: http://www.cdc.gov/ncidod/dvbid/westnile/ clinicians/.

2.Gubler DJ. The continuing spread of West Nile virus in the western hemisphere. Clin Infect Dis 2007;45:1039-1046.

3. Haley RW. Controlling urban epidemics of West Nile virus infection. JAMA 2012;308:13251326.

4. Vance K, Howe W, Dellavalle RP. Social Internet sites as a source of public health information. Dermatol Clin 2009;27:133-136.

5. USA Today. YouTube serves up 100 million videos a day online. Available at: http://usatoday30.usatoday.com/tech/news/200607-16-youtube-views_x.htm. Last accessed December 22, 2014.

6. Ache KA, Wallace LS. Human papillomavirus vaccination coverage on YouTube. Am J Prev Med 2008;35:389 -392.

7. Keelan J, Pavri-Garcia V, Tomlinson G, Wilson K. YouTube as a source of information on immunization: a content analysis. JAMA 2007;298:2482-2484.

8. Freeman B, Chapman S. Is "YouTube" telling or selling you something? Tobacco content on the YouTube video-sharing website. Tob Control 2007;16:207-210.

9. Eglash A. Website review:www.Youtube.com. Breastfeed Med 2009;4:119

10. Pandey A, Patni N, Singh M, Sood A, Singh G. YouTube as a source of information on the H1N1 influenza pandemic. Am J Prev Med 2010;38:e1-e3.

11. Sood A, Sarangi S, Pandey A, Murugiah K. YouTube as a source of information on kidney stone disease. Urology 2011;77:558-562.
12. Tourinho FS, de Medeiros KS, Salvador PT, Castro GL, Santos VE. Analysis of the YouTube videos on basic life support and cardiopulmonary resuscitation. Rev Col Bras Cir 2012;39:335-339. [Article in English, Portuguese].

13. Fat MJ, Sell E, Barrowman N, Doja A. Public perception of Tourette syndrome on YouTube. J Child Neurol 2012;27:1011-1016.

14. Kerber KA, Burke JF, Skolarus LE, Callaghan BC, Fife TD, Baloh RW, Fendrick AM. A prescription for the Epley maneuver: www.youtube.com? Neurology 2012;79:376-380.

15. Hartley DM, Barker CM, Le Menach A, Niu T, Gaff HD, Reisen WK. Effects of temperature on emergence and seasonality of West Nile virus in California. Am J Trop Med Hyg 2012;86:884-894.

\section{Author Affiliations}

Divyanshu Dubey, $M D^{*}$; Amod Amritphale, $M D^{\dagger}$; Anshudha Sawhney, MBBS"; Devashish Dubey, MBBS $S^{\S}$; Nupur Srivastav, MBBS

* Department of Neurology, University of Texas Southwestern, Dallas, TX, USA

${ }^{\dagger}$ Department of Internal Medicine, University of Nevada

School of Medicine, Las Vegas, NV, USA

${ }^{\star}$ Netaji Subhash Chandra Bose Medical College, Jabalpur, $M P$, India

${ }^{\S}$ Kasturba Gandhi Medical Center, Manipal, Karnataka, India

'Department of Pediatrics, Sir Ganga Ram Hospital, New Delhi, India 